\title{
Medication Adherence Interventions for Cardiovascular Disease in Low- and Middle-Income Countries: A Systematic Review
}

\author{
Oluwabunmi Ogungbe $\mathbb{D}^{\prime}$ \\ Samuel Byiringiro (D) \\ Adeola Adedokun- \\ Afolayan (D) ${ }^{2}$ \\ Stella M Seal $\mathbb{D}^{3}$ \\ Cheryl R Dennison \\ Himmelfarb (D) ${ }^{1,4,5}$ \\ Patricia M Davidson (D) \\ Yvonne Commodore- \\ Mensah (ID) ${ }^{1,5}$ \\ 'Johns Hopkins University School of \\ Nursing, Baltimore, MD, USA; ${ }^{2}$ School of \\ Public Health, Louisiana State University, \\ Shreveport, LA, USA; ${ }^{3}$ William H. Welch \\ Medical Library, Johns Hopkins University \\ and Medicine, Baltimore, MD, USA; ${ }^{4}$ Johns \\ Hopkins University School of Medicine, \\ Baltimore, MD, USA; ${ }^{5}$ Department of \\ Epidemiology, Johns Hopkins Bloomberg \\ School of Public Health, Baltimore, MD, USA
}

Correspondence: Oluwabunmi Ogungbe Johns Hopkins University School of Nursing, $525 \mathrm{~N}$. Wolfe Street, Baltimore, MD, 21205, USA

Tel + I-60I-54I-I I52

Email oogungb3@jh.edu
Purpose: The burden of cardiovascular diseases (CVD) is high in low- and middle-income countries (LMICs). Medications are integral to the management and control of CVD; however, suboptimal adherence impacts health outcomes. This systematic review aims to critically examine interventions targeted at improving medication adherence among persons with CVD in LMICs.

Methods: In this systematic review, we searched online databases PubMed, Embase, and CINAHL for studies that evaluated a medication adherence intervention for CVD, reported adherence as an outcome measure, were conducted in LMICs and reported the strategy or tool used to measure adherence. We included articles published in English, available in full text, peer-reviewed, and published between 2010 and 2020.

Results: We included 45 articles in this review. The majority of the studies implemented counseling and educational interventions led by nurses, pharmacists, or community health workers. Many of the studies delivered medication-taking reminders in the form of phone calls, text messages, short message services (SMS), and in-phone calendars. Multi-component interventions were more effective than unifocal interventions. Interventions involving technology, such as mobile phone calls, electronic pillboxes, and interactive phone SMS reminders, were more effective than generic reminders. The outcomes reported in the studies varied based on the complexity and combination of strategies. When interventions were implemented at both the patient level, such as reminders, and at the provider level, such as team-based care, the effect on medication adherence was larger.

Conclusion: In LMICs, medication adherence interventions among persons with CVD included a combination of patient education, reminders, fixed-dose combination therapy and team-based care approach were generally more effective than singular interventions. Among patients who had CVD, the medication adherence interventions were found to be moderately effective. Future studies focusing on improving medication adherence in LMICs should consider non-physician-led interventions and appropriately adapt the interventions to the local context.

Keywords: medication adherence, cardiovascular diseases, LMICs, systematic review

\section{Introduction}

Cardiovascular disease (CVD) is the leading cause of morbidity and mortality globally, accounting for about 17 million (30\%) deaths annually. ${ }^{1}$ This number of CVD deaths is projected to increase to over 23.3 million by $2030 .{ }^{1}$ The population most affected are people living in regions where more than $80 \%$ of all CVD deaths occur. ${ }^{2}$ Although the CVD epidemic has begun to recede in some high-income countries (HICs), CVD mortality rates in low- and middle-income countries (LMICs) continue to rise to 
about 300-600 CVD deaths per 100,000 population every year. Of note, in countries such as the United States, some of the gains achieved are being lost. ${ }^{1}$ Sub-optimal adherence to medications for the prevention and treatment and chronic conditions is considered a significant public health concern. It is also associated with poor control of CVD risk factors, CVD complications, worse health outcomes, and increased healthcare costs. ${ }^{3,4}$ In HICs, optimal adherence is only about $50 \%$ among patients who have CVD. Adherence to CVD medications is even lower in emerging economies where there are challenges of limited health resources, socioeconomic barriers, and inequities in access to healthcare. ${ }^{3,5}$

Adherence is defined as the extent to which a person's medication-taking behavior corresponds with an agreed recommendation from a healthcare provider. $^{6}$ Achieving $80 \%$ or higher adherence to recommendations is considered "good", 7,8 The treatment of CVD usually involves long-term use of medications, and their full benefit is often undetected as only about $50 \%$ of patients take their medications as prescribed. ${ }^{9}$ Barriers to medication adherence include forgetfulness, cost, side effects, cultural beliefs, health insurance, depression, comorbidities, polypharmacy, lack of social support, patient-provider communications and relationships, and lack of health insurance. ${ }^{10,11}$

There are several interventions for improving medication adherence: patient education, medication regimen management, fixed-dose combination medications, consultation with clinical pharmacists, and team-based care. ${ }^{12,13}$ Other strategies are cognitive-behavioral therapies, use of incentives, and medication-taking reminders such as electronic pill monitoring with text messages, automated refill tracking of in-patient electronic records, or email alerts to a provider for missed refills. ${ }^{12}$ While these strategies have been widely used in research and healthcare practice in high-income countries; they have not been sufficiently adapted for use in LMICswhere the burden of diseases is high, and challenges with medication utilization are higher. ${ }^{13,14}$ It has been suggested that increases in medication adherence interventions would likely have a more significant impact on the health of the population than other specific medication treatments. ${ }^{3}$ While studies have described medication adherence as being low in LMICs and focused on the barriers and factors influencing, research is scarce regarding the implementation of medication adherence strategies in these settings. ${ }^{14,15}$ Therefore, this study aimed to critically examine interventions targeted at improving medication adherence among persons with cardiovascular diseases in LMICs.

\section{Methods}

\section{Search Strategy and Selection Criteria}

Using recommendations from the Preferred Reporting Items for Systematic Reviews and Meta-Analysis (PRISMA) ${ }^{16}$ and with the help of an information specialist, we conducted a literature review on medication adherence interventions for cardiovascular diseases in LMICs. We built a search strategy using relevant text words and their synonyms (Table S1); we also searched controlled vocabulary in the databases: Emtree in Embase, MeSH in PubMed, and subject headings Cumulative Index to Nursing \& Allied Health Literature (CINAHL). Final searches were conducted on August 11, 2020, in PubMed, Embase, and CINAHL. The final search strategy can be found in the Supplemental Files (Table S1). We imported identified articles into Covidence $^{\circledR 17}$ and titles and abstracts were screened for eligibility based on the inclusion and exclusion criteria described below. We included studies that implemented or tested a medication adherence intervention for cardiovascular diseases, reported adherence as an outcome measure, were conducted in LMICs, and reported the strategy or tool used to measure adherence. The articles had to be published in English, available in full text, peer-reviewed, and published between 2010 and 2020. Studies that implemented medication adherence in conditions other than CVD were excluded. Systematic reviews, study protocols, editorials, and commentaries were excluded, including low-quality articles appraised using the Joanna Briggs Institute (JBI) Critical Appraisal Tools (Table S2). ${ }^{18}$ Following the screening of titles and abstracts, full-text versions of screened articles were obtained. Two authors (B.O. and S.B.) independently reviewed the full text articles to determine the studies' eligibility and subsequently extracted the data. During the fulltext review process, discrepancies and disagreements were resolved through discussion and review by a third, independent author (A.A.). The PRISMA checklist and flowchart were also used to facilitate transparent reporting of the articles reviewed. ${ }^{16}$ The review protocol was registered in PROSPERO with registration number CRD42020211279.

\section{Results}

A total of 45 studies that met our inclusion criteria were included in this review (Figure 1). Four studies were conducted in Africa: two in Nigeria, ${ }^{19,20}$ one in Ghana, ${ }^{21}$ and one in South Africa ${ }^{22}$ Eight of the studies were conducted in the Americas: Brazil, ${ }^{23-27}$ Argentina, ${ }^{28}$ Portugal, ${ }^{29}$ and Chile. ${ }^{30}$ Thirty-three of the studies were conducted in Asia: 


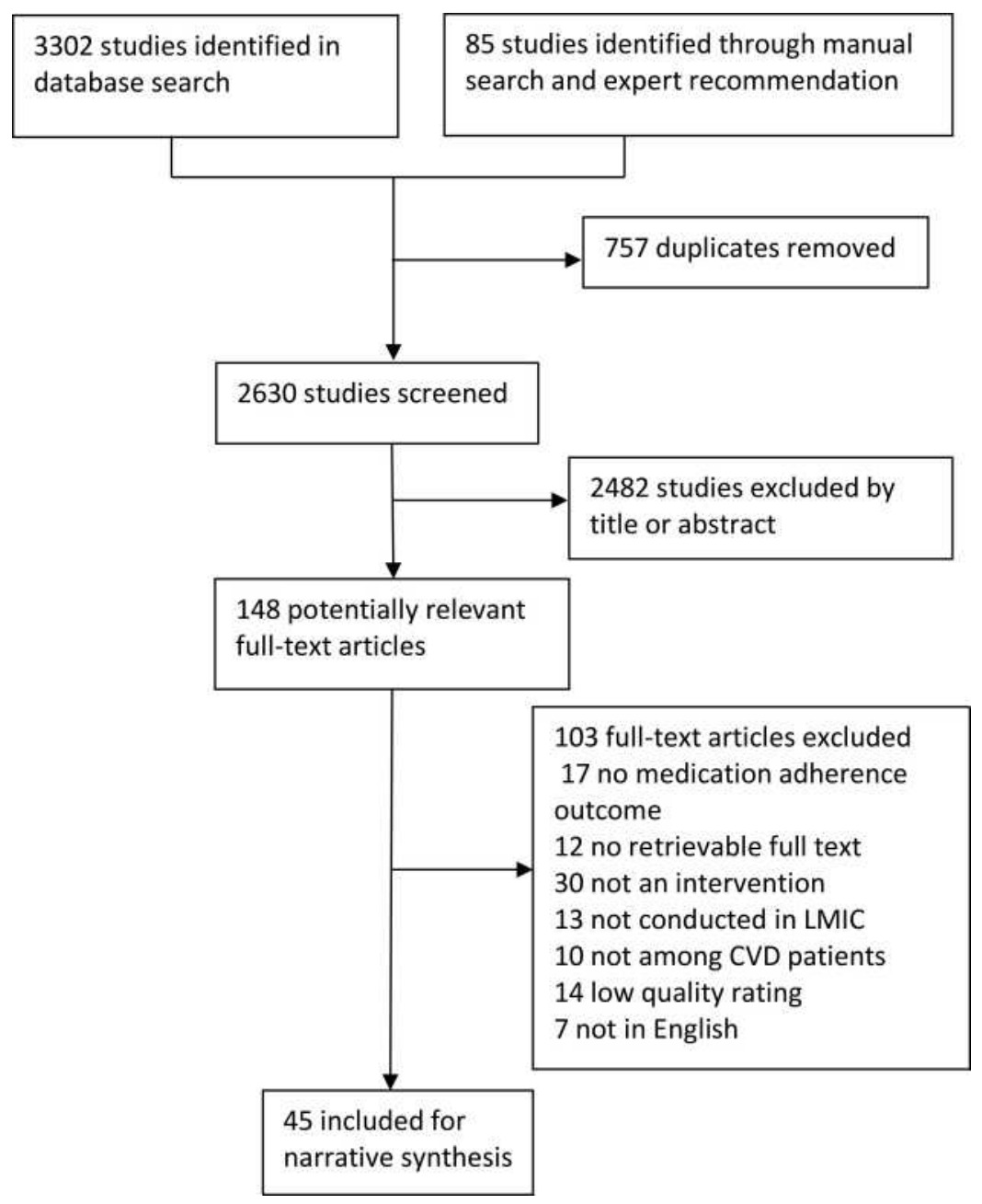

Figure I PRISMA flowchart showing the selection of eligible studies.

Note: Copied from Moher D, Liberati A, Tetzlaff J, Altman DG, The PRISMA Group (2009). Preferred Reporting Items for Systematic Reviews and Meta-Analyses: The PRISMA Statement. PLoS Med 6(7): el $000097 .{ }^{16}$

Jordan, ${ }^{31,32}$ Iran, ${ }^{33-41}$ Philippines, ${ }^{42}$ Malaysia, ${ }^{43}$ China, ${ }^{44-50}$ Taiwan, ${ }^{51}$ India, ${ }^{52-57}$ Vietnam, ${ }^{58}$ Pakistan, ${ }^{59-61}$ and Thailand $^{62,63}$ (Table 1). Also, 35 of the studies were randomized clinical trials and nine articles were non-randomized studies; one study was a cohort study; others were quasiexperimental and pre-post studies. The sample size of the studies included in the review ranged from 30 to 5725 . The total population in the intervention groups across all the studies was 25,493; the mean was 554 participants. For all the control groups, the total participants were 6315; the mean was 162 participants. The duration of interventions in the studies ranged from 4 weeks to 12 months.

In this review, many of the studies included multiple interventions that contributed to a more substantial effect on medication adherence. Almost three-fourths (72\%, $\mathrm{n}=33$ ) of the studies used a multi-component approach to the interventions. The complexity level of the interventions did not necessarily translate into a stronger effect. The dimensions of medication adherence determinants were provider, drug or therapy-level, and health system-level factors. Thus, medication adherence interventions were classified as patient, provider, drug/therapy, and health system-level interventions. Majority $(91 \%, n=41)$ of the studies included in this review addressed medication adherence at the patient level. These interventions included fixed-dose combination therapy, patient education, lifestyle counseling, cognitive behavioral therapy, reminders, and incentives.

When educational interventions were customized, initiated early, and repeated at regular intervals, improvements in medication adherence were shown to be modest; $73 \%(n=33)$ of the interventions that included patient education were effective. The most substantial effect size 


\begin{tabular}{|c|c|c|c|c|c|c|c|c|c|c|c|}
\hline 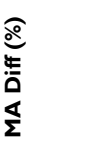 & & & $\begin{array}{c}\text { to } \\
\text { II. } \\
\text { II } \\
\text { ô }\end{array}$ & $\begin{array}{l}\text { on } \\
\text { ò } \\
\text { +1 } \\
\text { d } \\
0\end{array}$ & $\begin{array}{l}\frac{\tilde{n}}{\ddot{n}} \\
\dot{\tilde{o}}\end{array}$ & 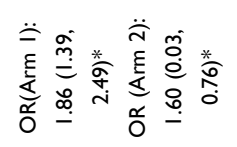 & & 器 & 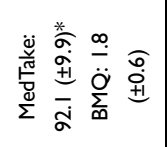 & $\begin{array}{l}\stackrel{*}{*} \\
\underline{\infty}\end{array}$ & 萰 \\
\hline 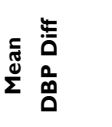 & & & 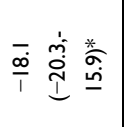 & $\mathbb{Z}$ & $\mathbb{z}$ & $\stackrel{\alpha}{z}$ & & 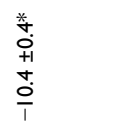 & $\stackrel{\alpha}{z}$ & 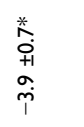 & 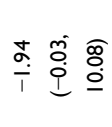 \\
\hline 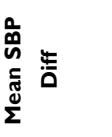 & & & 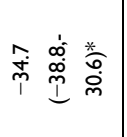 & $\mathbb{z}$ & $\mathbb{z}$ & 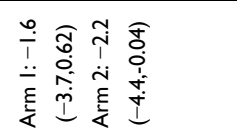 & & 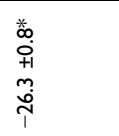 & $\stackrel{\alpha}{Z}$ & 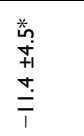 & 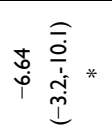 \\
\hline 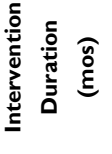 & & & 0 & $m$ & 旁 & $\simeq$ & & $\underline{\circ}$ & $=$ & 0 & N \\
\hline 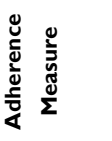 & & & 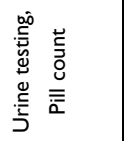 & $\frac{\alpha}{\Sigma}$ & $\begin{array}{l}\infty \\
\sum_{i}^{\frac{T}{\Sigma}} \\
\Sigma\end{array}$ & $\sum_{0}^{U}$ & & $\frac{n}{\Sigma}$ & 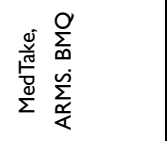 & $\sum_{\infty}^{\frac{O}{\infty}}$ & $\begin{array}{l}\frac{I}{5} \\
\frac{5}{\delta}\end{array}$ \\
\hline ¿̀̃ & & & 站 & $\begin{array}{l}\stackrel{0}{\underline{\underline{\nu}}} \\
\text { ż }\end{array}$ & $\begin{array}{l}\text { 品 } \\
\text { ż }\end{array}$ & 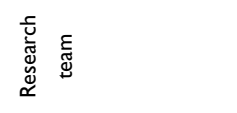 & & $\frac{E}{\frac{E}{\underline{\underline{n}}}}$ & $\underset{\frac{E}{\tilde{c}}}{\frac{E}{\alpha}}$ & $\frac{E}{\frac{E}{\frac{\pi}{a}}}$ & 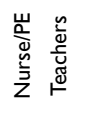 \\
\hline & 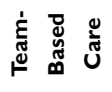 & & + & + & & & & + & + & & \\
\hline & 竘 & & + & & & & $\tilde{g}$ & & & & \\
\hline إِ & 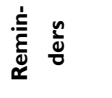 & $\frac{z}{4}$ & & + & & + & 离 & & + & & \\
\hline$\underline{\underline{E}}$ & U. & & & & & & & & & & \\
\hline & 备 & & & & & & & & & & \\
\hline & ż & & + & & + & + & & + & + & + & + \\
\hline & Do & & 志总高亳 & 只总旁 & $\mathbb{z}$ & 字昜 & & $\mathbb{Z}$ & $\tilde{n}$ & $\begin{array}{l}\frac{n}{5} \\
\frac{5}{5} \\
0 \\
\text { m }\end{array}$ & $\underline{z}$ \\
\hline Е్ & 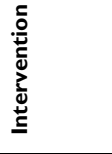 & & 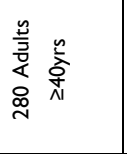 & 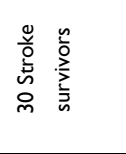 & $\stackrel{g}{\underline{G}}$ & 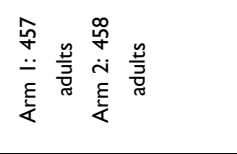 & & 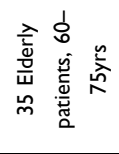 & $\bar{n}$ & $\begin{array}{l}\frac{n}{5} \\
\frac{5}{5} \\
\tilde{m}\end{array}$ & $\begin{array}{l}\frac{\underline{\underline{n}}}{\bar{J}} \\
\stackrel{0}{0} \\
\underline{0}\end{array}$ \\
\hline 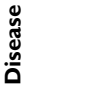 & & & 圣 & $\begin{array}{l}\stackrel{0}{\circ} \\
\stackrel{0}{5}\end{array}$ & 己 & 㝊 & & 圣 & 己 & $\stackrel{\mathscr{y}}{\Sigma}$ & 胥 \\
\hline 高 & & & $\underset{\alpha}{\mathfrak{s}}$ & 䇏 & 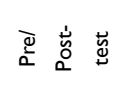 & 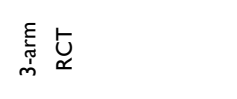 & & す & 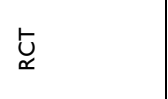 & $\underset{\longleftarrow}{t}$ & $\begin{array}{l}\overline{\bar{m}} \\
\bar{z}\end{array}$ \\
\hline نे & & & 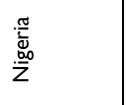 & $\begin{array}{l}\frac{\pi}{\tilde{J}} \\
\frac{\pi}{0}\end{array}$ & 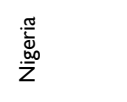 & 吾蒙 & & $\overline{\overline{\bar{x}}}$ & $\overline{\overline{\bar{N}}}$ & 胥 & 突 \\
\hline 蒿 & & & 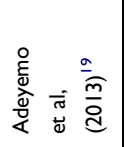 & 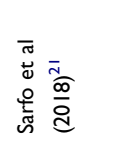 & 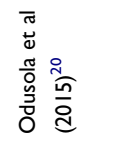 & 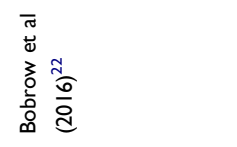 & & 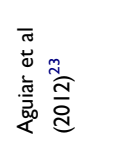 & 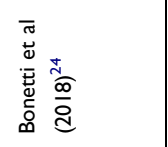 & 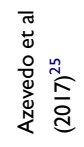 & 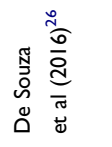 \\
\hline
\end{tabular}




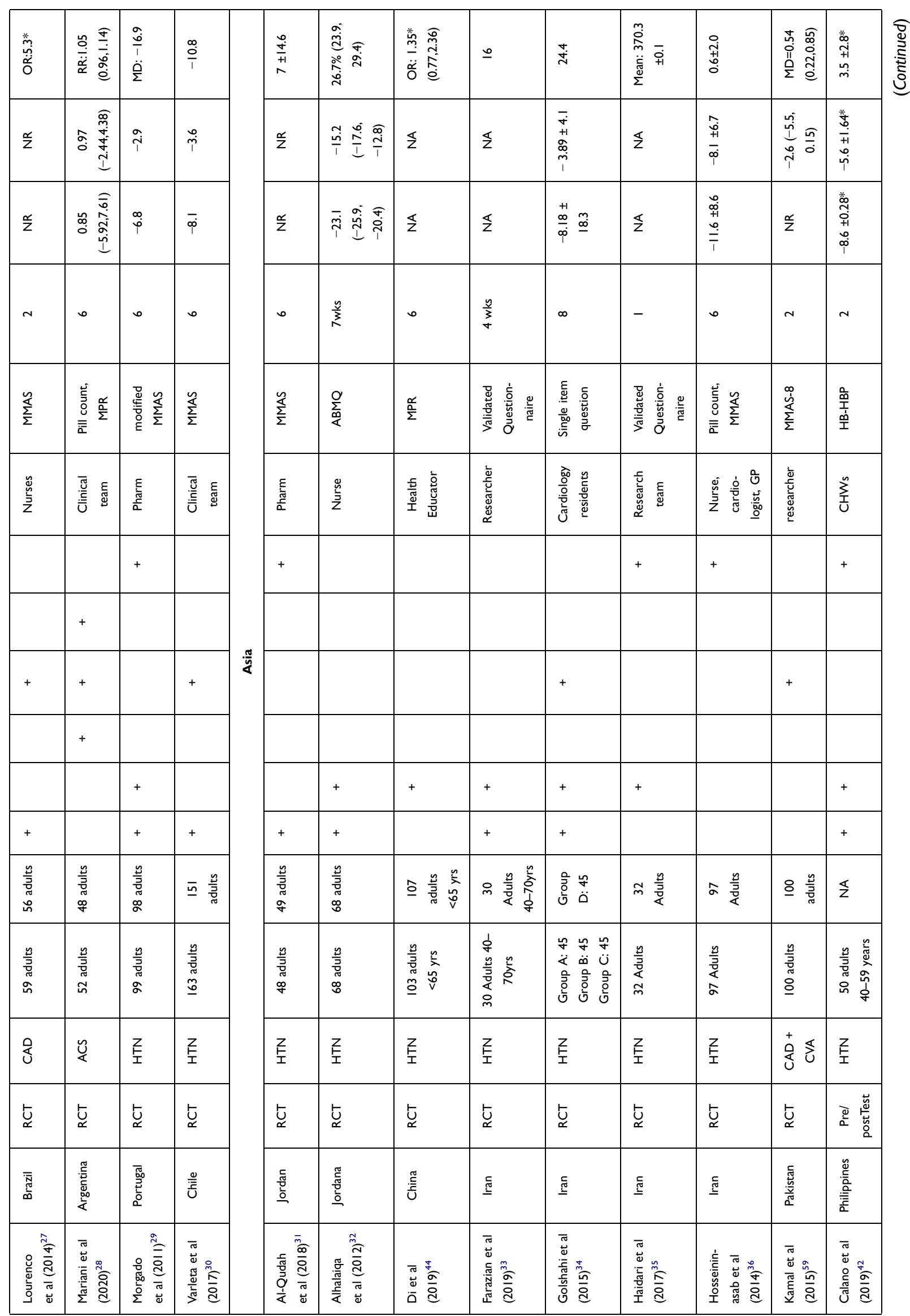




\begin{tabular}{|c|c|c|c|c|c|c|c|c|c|c|}
\hline 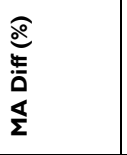 & & 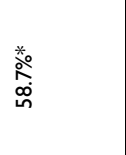 & 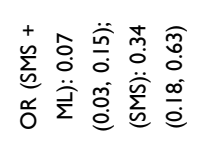 & 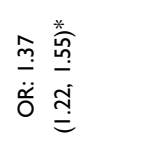 & 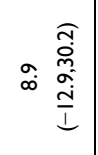 & $†$ & $\stackrel{\stackrel{n}{\varphi}}{\varphi}$ & 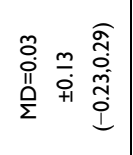 & 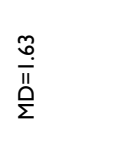 & 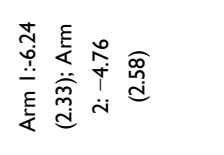 \\
\hline 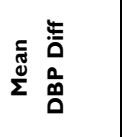 & & 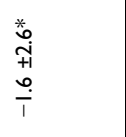 & $\mathbb{z}$ & $\mathbb{z}$ & 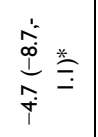 & $\stackrel{\mathscr{\alpha}}{\mathrm{z}}$ & $\stackrel{\mathscr{N}}{\mathrm{z}}$ & $\frac{\alpha}{z}$ & 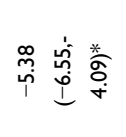 & $\stackrel{\mathscr{L}}{\mathrm{z}}$ \\
\hline 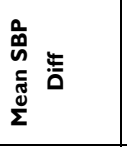 & & 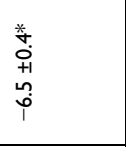 & $\frac{\pi}{z}$ & $\mathbb{z}$ & 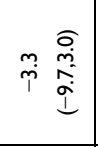 & 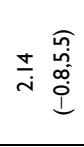 & $\begin{array}{l}0 \\
\stackrel{0}{+} \\
\text { +1 } \\
\text { i }\end{array}$ & $\stackrel{\mathscr{\alpha}}{Z}$ & 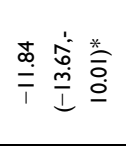 & $\stackrel{\alpha}{z}$ \\
\hline 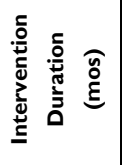 & & $\sigma$ & $m$ & ○ & $\stackrel{n}{3}$ & $\circ$ & $\simeq$ & $m$ & $\circ$ & $m$ \\
\hline 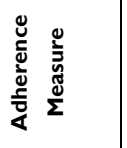 & & 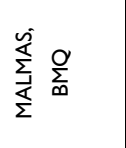 & $\sum_{\Sigma}^{\frac{U}{\Sigma}}$ & $\frac{\alpha}{\Sigma}$ & 岕 & 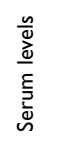 & $\frac{U}{\sum_{L}}$ & $\begin{array}{l}\infty \\
\substack{\vec{T} \\
\Sigma} \\
\sum\end{array}$ & $\frac{n}{\Sigma}$ & $\begin{array}{l}\text { 譬 } \\
\text { 产 }\end{array}$ \\
\hline$\frac{\grave{U}}{\mathfrak{I}}$ & & $\underset{\frac{E}{d \frac{E}{c}}}{\frac{\varepsilon}{c}}$ & 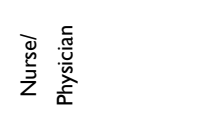 & 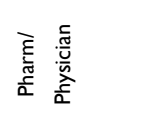 & 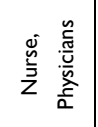 & 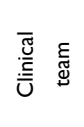 & 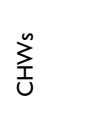 & 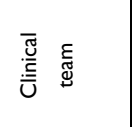 & 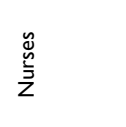 & 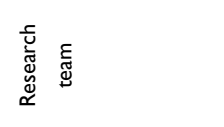 \\
\hline \multirow{6}{*}{ 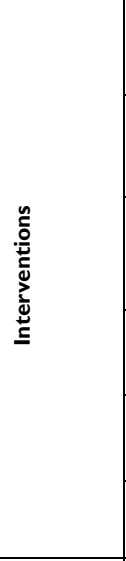 } & 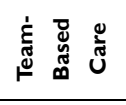 & & & & & & + & & + & \\
\hline & 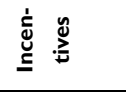 & & & & & & & & & \\
\hline & 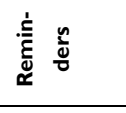 & & + & & & + & & + & + & + \\
\hline & Ŭ & & & + & & & & & & \\
\hline & 总 & & & & + & & & + & & \\
\hline & 胥 & + & + & & + & & + & & & + \\
\hline \multirow{2}{*}{ 造 } & 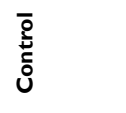 & $\mathbb{z}$ & $\ddot{\sigma}$ & 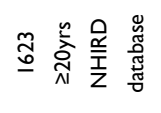 & $\neq \frac{\frac{n}{z}}{\frac{z}{\alpha}}$ & $\overline{\check{~}}$ & $\stackrel{g}{\underline{\underline{g}}} \frac{\underline{y}}{\bar{z}}$ & 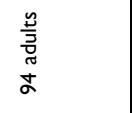 & 品 亭 & 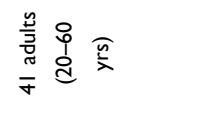 \\
\hline & 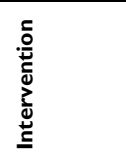 & $\begin{array}{l}\frac{n}{5} \\
\frac{5}{5} \\
\forall \\
\forall\end{array}$ & 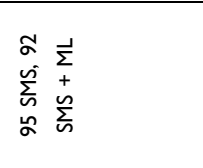 & 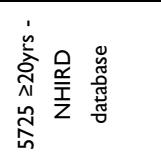 & 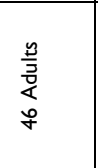 & $\begin{array}{l}\frac{0}{\overline{5}} \\
\frac{i}{\sqrt{n}}\end{array}$ & 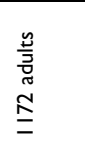 & $\begin{array}{l}\frac{n}{5} \\
\frac{5}{\sigma} \\
\sigma\end{array}$ & 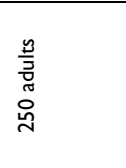 & 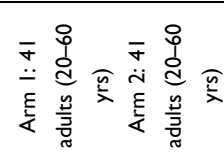 \\
\hline 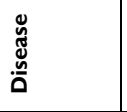 & & 罕 & 守 & 罕 & 오 & 享 & 罕 & 离苍 & O & 罕 \\
\hline$\frac{.50}{\bar{g}}$ & & 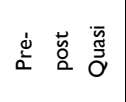 & 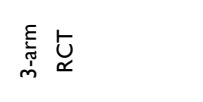 & $\begin{array}{l}\frac{1}{0} \\
\frac{0}{0} \\
\text { uे }\end{array}$ & 产 & $\stackrel{t}{\simeq}$ & $\begin{array}{l}\bar{y} \\
\text { 产 } \\
\bar{u}\end{array}$ & $\underset{\longleftarrow}{\longleftarrow}$ & 离 & $\underset{\check{c}}{\grave{1}}$ \\
\hline نे & & 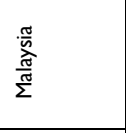 & $\frac{\widetilde{g}}{\tilde{U}}$ & 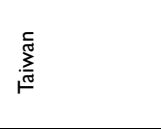 & $\underset{\mathscr{E}}{\tilde{E}}$ & 㞼 & $\stackrel{\frac{\pi}{\tilde{g}}}{\underline{\underline{g}}}$ & $\frac{\sqrt{5}}{\frac{5}{\frac{5}{0}}}$ & $\underline{\underline{\underline{\underline{g}}}}$ & 胥 \\
\hline 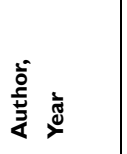 & & 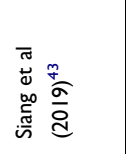 & 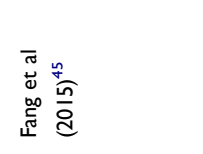 & 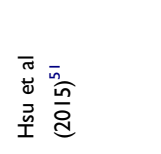 & 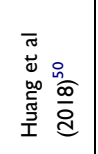 & 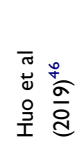 & 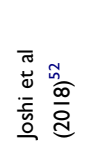 & 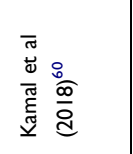 & 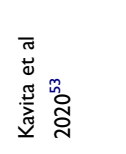 & 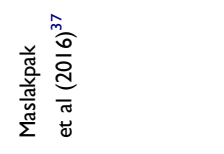 \\
\hline
\end{tabular}




\begin{tabular}{|c|c|c|c|c|c|c|c|c|c|c|}
\hline 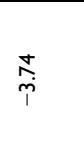 & 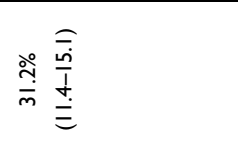 & 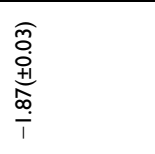 & 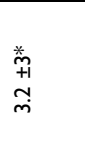 & $\stackrel{\frac{*}{m}}{i}$ & 并 & $\underset{i}{\stackrel{*}{*}}$ & 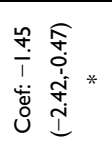 & $\frac{*}{\sigma}$ & 晜 & 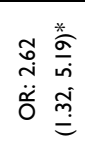 \\
\hline 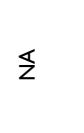 & 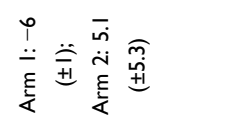 & $\stackrel{\alpha}{z}$ & $\dot{p}$ & $\begin{array}{l}\overline{\bar{c}} \\
+1 \\
+\vdots \\
\dot{i} \\
\text { i }\end{array}$ & $\begin{array}{l}\stackrel{0}{0} \\
\stackrel{+}{+1} \\
\stackrel{\varphi}{r}\end{array}$ & 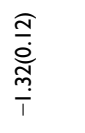 & $\mathbb{Z}$ & 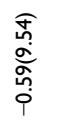 & 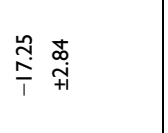 & $\begin{array}{l}\text { ñ } \\
\text { +1 } \\
o \\
\text { ò }\end{array}$ \\
\hline 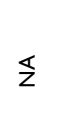 & 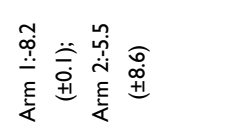 & $\stackrel{\alpha}{z}$ & $\uparrow$ & 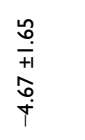 & 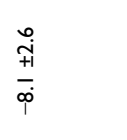 & 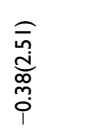 & $\underline{z}$ & 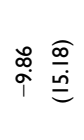 & 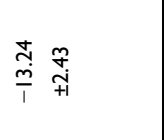 & $\begin{array}{l}\stackrel{+}{+} \\
+1 \\
\stackrel{+}{0}\end{array}$ \\
\hline$m$ & $m$ & $N$ & ${ }^{\circ}$ & $\circ$ & $\stackrel{ \pm}{\sim}$ & $\circ$ & $\circ$ & $m$ & $m$ & $\simeq$ \\
\hline$\sum_{\sum}^{u}$ & 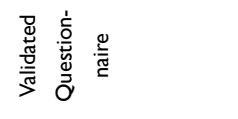 & $\begin{array}{l}\frac{\infty}{\sum_{\alpha}} \\
\dot{\alpha} \\
\text { 安 }\end{array}$ & $\frac{0}{\frac{1}{\Delta}}$ & $\frac{\mathscr{c}}{\Sigma}$ & 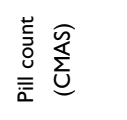 & $\frac{\infty}{\sum_{L}^{N}}$ & $\begin{array}{l}\stackrel{0}{\underline{m}} \\
\frac{\underline{m}}{\underline{1}}\end{array}$ & $\begin{array}{l}= \\
\bar{a} \\
\frac{a}{1}\end{array}$ & $\begin{array}{l}\frac{\breve{s}}{\stackrel{\bar{o}}{0}} \\
\overline{\overline{\bar{\alpha}}}\end{array}$ & 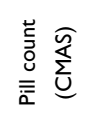 \\
\hline 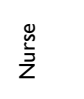 & $\sum_{\text {S }}^{n}$ & 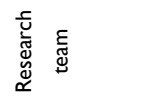 & $\frac{E}{\frac{E}{0}}$ & $\frac{E}{\frac{E}{0}}$ & 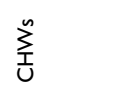 & 竞 & $\sum_{\text {I }}^{n}$ & 总 & 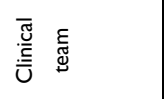 & $\sum_{\text {S }}^{n}$ \\
\hline & & + & & & & & & & + & + \\
\hline & & & & & & & & & + & \\
\hline+ & & & & & + & + & + & + & + & + \\
\hline & & & & & & & & & + & \\
\hline & + & & + & & & & + & + & & \\
\hline+ & + & & + & + & + & + & + & + & + & + \\
\hline $\begin{array}{l}\frac{y}{3} \\
\frac{5}{\tilde{z}} \\
\text { in }\end{array}$ & $\stackrel{\alpha}{Z}$ & 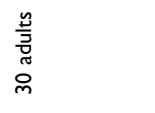 & $\stackrel{\sigma}{\frac{g}{3}}$ & 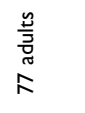 & 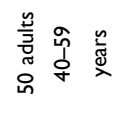 & $\begin{array}{l}\frac{n}{5} \\
\frac{5}{5} \\
\stackrel{0}{\infty}\end{array}$ & $\underline{z}$ & $\begin{array}{l}\frac{n}{5} \\
\bar{J} \\
\infty \\
\infty\end{array}$ & 으 蒙 & $\stackrel{n}{n} \frac{y}{y}$ \\
\hline 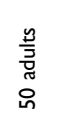 & 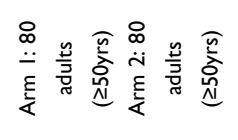 & 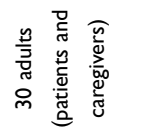 & $\begin{array}{l}\underline{\underline{n}} \\
\underline{\underline{z}} \\
\underline{\underline{m}}\end{array}$ & $\begin{array}{l}\frac{n}{5} \\
\frac{\tilde{g}}{\pi} \\
\kappa\end{array}$ & 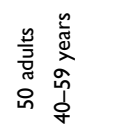 & $\begin{array}{l}\frac{n}{5} \\
\frac{5}{0} \\
\infty\end{array}$ & 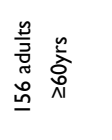 & $\begin{array}{l}\frac{n}{3} \\
\frac{\tilde{z}}{\tilde{J}} \\
\hat{\infty}\end{array}$ & 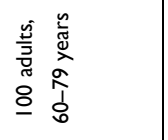 & $\begin{array}{l}\frac{n}{5} \\
\text { 言 } \\
\stackrel{n}{m} \\
\text { m }\end{array}$ \\
\hline $\bar{\Sigma}$ & U & 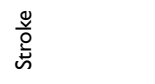 & 杢 & $\bar{\Sigma}$ & $\dddot{u}$ & 埾 & 宔 & 辛总 & 圣 & $\ddot{\psi}$ \\
\hline$\stackrel{5}{\simeq}$ & 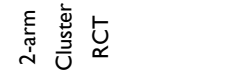 & 离苅 合 & 商 & 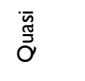 & $\underset{\check{c}}{\mathfrak{x}}$ & $\underset{\longleftarrow}{\longleftarrow}$ & 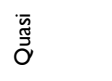 & $\underset{c}{\mathfrak{g}}$ & 产 & $\stackrel{5}{x}$ \\
\hline$\underline{\underline{\mathbb{E}}}$ & 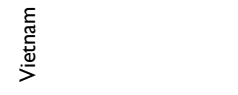 & $\underline{\underline{\underline{\underline{e}}}}$ & 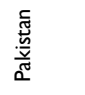 & 吾 & 営 & 営 & 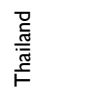 & 晃 & 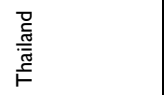 & 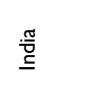 \\
\hline 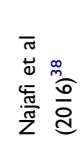 & 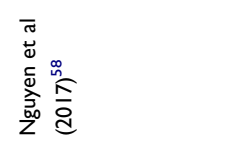 & 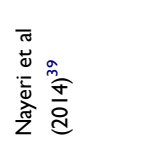 & 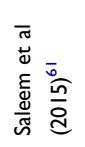 & 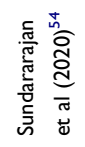 & 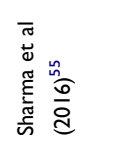 & 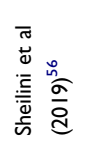 & 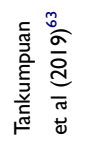 & 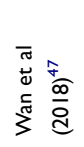 & 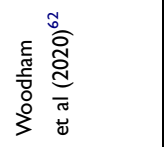 & 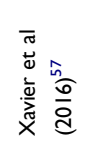 \\
\hline
\end{tabular}




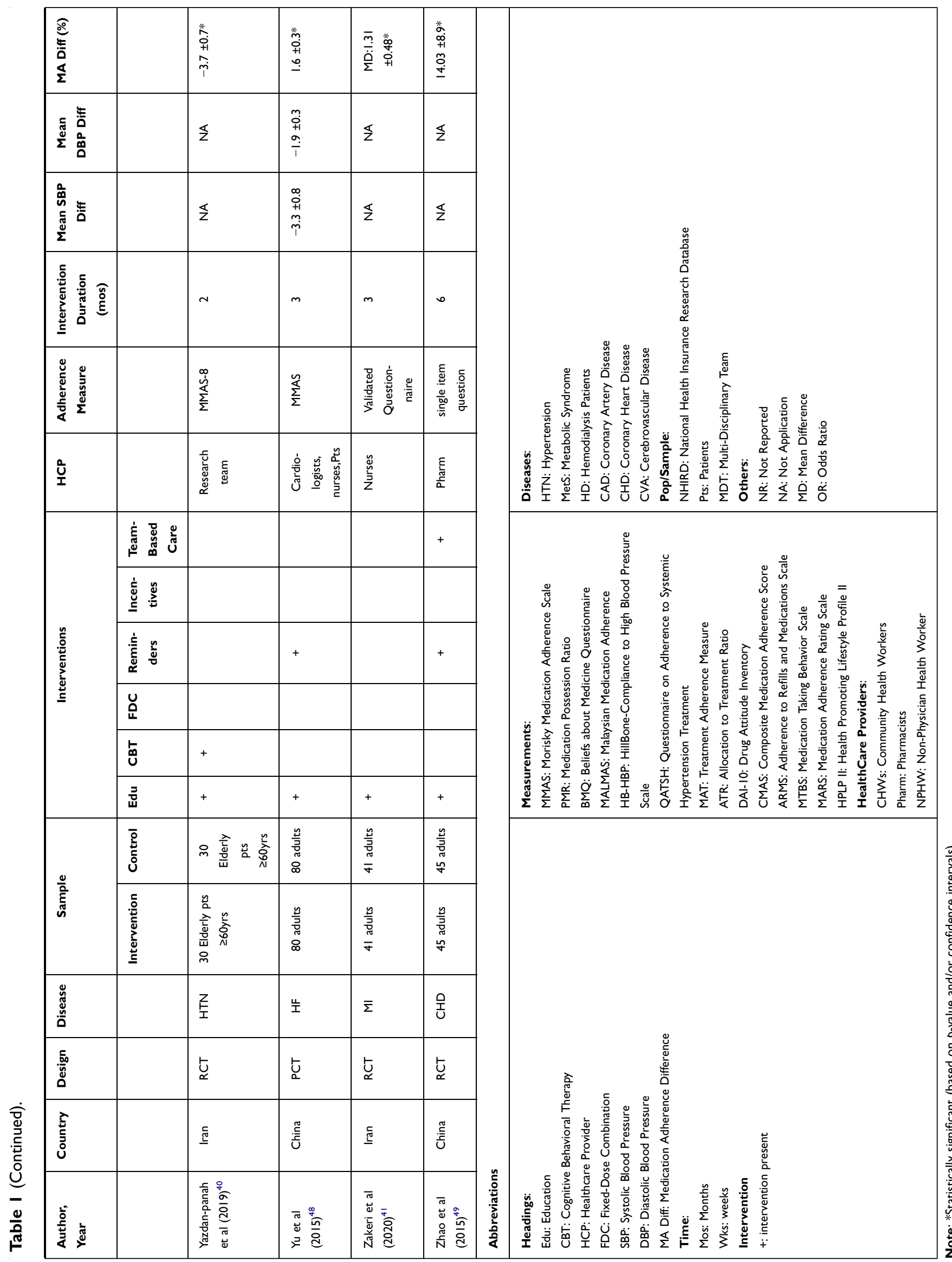


was observed in Lourenco et al, ${ }^{27}$ a nurse-led intervention of in-person visits and made plans on medication-taking behavior with phone reinforcements. Medication adherence was more improved in the intervention group than in the control group after two months of follow-up (OR: 5.23, 95\% CI: $2.03-13.49 ; p=0.001$ ). The smallest effect size was observed in Kamal et al, ${ }^{59}$ where the intervention group received daily interactive voice calls regarding their medications for stroke and myocardial infarction (MI), daily tailored medication reminders, and weekly lifestyle modifications for three months. At the end of follow-up, the mean medication adherence was increased in the intervention group compared with the usual care group with a mean difference of $0.03( \pm 0.13)$, (95\% C.I: $-0.23-0.29 ; p=$ $0.40)$. Nurses provided education in $41 \%(n=19)$ of the studies, including as part of the clinical team; physicians provided education in $27 \%(\mathrm{n}=12)$ of the studies, pharmacists provided education in $22 \%(\mathrm{n}=10)$ of the studies, community health workers provided education in $13 \%$ $(n=6)$ of the studies. The duration of the education was brief in some cases and delivered in a single session, while in other instances, education was delivered multiple times. Interventions in which patient education was delivered in-person, face-to-face were more likely to have a higher effect on medication adherence.

Medication-taking reminders of phone calls, text messages, Short Message Services (SMS), or in-phone calendars were some of the most common medication adherence interventions. Reminders were more effective when they were personalized or interactive rather than generic. Many $(48 \%$, $\mathrm{n}=22)$ of the interventions included in this review were conducted by phone calls $\mathrm{s}^{24,33,45,48,49,57,64,65}$ or by SMSs, including customized and interactive messages ${ }^{30,45,46,60}$ and electronic pillboxes. ${ }^{62}$ Only one study implemented incentives as a strategy to improve adherence in the form of free antihypertensive medication and transportation funds to attend clinic appointments. ${ }^{19}$ In the management of chronic diseases, a team-based approach, or team-based care, was identified as a strategy that may improve adherence. In this review, the interventions incorporated a team-based approach to CVD management and medication adherence. These interventions were nurse-led, ${ }^{19,21,26,27,32,36,38,41,45,48,53,56}$ community health worker-led, ${ }^{52,55,57}$ and clinical/community pharmacistled. ${ }^{23-25,31,43,61,62}$ In Kavita et $\mathrm{al}^{53}$ a team-based approach was used to deliver a medication adherence intervention; a group of experts from cardiology, nursing, community medicine, and fine arts developed and validated an intervention package that consisted of a booklet for nurses, a patient education booklet and flashcards for patient education. After one year of follow-up, the mean adherence scores were significantly higher in the intervention group $(p<0.001)$; effect size (Cohen's d) was 1.1.

Fixed-dose combination therapy or single-dose therapy has been recommended for use in the initial treatment of CVD and CVD risk factors rather than monotherapy because they may facilitate long-term adherence. Mariani et $\mathrm{al}^{28}$ investigated whether a multi-cap containing four secondary prevention drugs would increase the adherence to treatment at six months following MI hospitalization and found that $98 \%$ of those who received the multi-cap were adherent to treatment six months after the intervention compared to $93.5 \%$ in the control group (RR: 1.05 ; $95 \%$ CI: $0.96-1.14 ; p=0.347)$; however, there were no significant improvements in medication adherence between the groups.

Indirect adherence measurement methods were the most common methods used in the articles reviewed (Table S3). These included the use of measurement scales, pharmacy chart records, self-report, pill counts, and calculating the medication possession ratio. Urine and blood testing were among the direct methods of assessment used in some of the studies. The measurement scales of medication adherence were among the most common and cost-effective ways of measuring medication adherence. These are validated scales, with acceptable reliability commonly used in research and clinical settings.

\section{Discussion}

This systematic review critically examined interventions targeted at improving medication adherence among patients with CVD in LMICs. Hypertension was the most common cardiovascular condition addressed across the studies. Interventions that were more effective at improving medication adherence included changing from multi-dose medications to fixed-dose combinations, team-based healthcare, ${ }^{31,33,53}$ and patient education combined with reminders. We also observed that studies that combined multiple medication adherence strategies in the interventions reported significant improvements in medication adherence. ${ }^{19,23,24,64}$ Our review builds on existing literature regarding medication adherence and highlights the medication adherence interventions conducted in LMIC.

Several factors contributed to non-adherence to CVD medications in LMICs. The extent of medication adherence was expected to be lower in LMIC due to a weaker health infrastructure and inequality in access to health care. These factors were outlined in the WHO report on adherence to long-term 
therapy and were also highlighted in a recent review of medication adherence in LMICs. ${ }^{3}$ Socioeconomic factors were significant contributors to medication non-adherence in LMICs, including long distances from treatment settings, high cost of medicines and limited drug supply, lower health literacy, family size, local beliefs about the origin of illnesses, and concerns about medical cost. ${ }^{3,12}$ Health care and systemrelated factors contributed significantly to non-adherence in LMICs, including inadequate or non-existent reimbursement by health insurance plans, irregular and insufficient drug supply, lack of medical supplies, poorly developed healthcare services, lack of knowledge and training for healthcare providers regarding managing CVD and other chronic diseases, lack of clear instructions from healthcare professionals including poor implementation of educational interventions. ${ }^{12}$

Healthcare resources are scarce in low- and middleincome countries, and the feasibility of interventions is hinged on their cost-effectiveness and focus on quality improvement. Medication adherence is considered multidimensional, and interventions that address patient-related factors alone have not shown long-term evidence of medication adherence improvements. ${ }^{66,67}$ Medication adherence interventions that are multifaceted are encouraged in LMICs because they present an opportunity to improve cardiovascular outcomes while reducing healthcare spending and maximizing the use of already limited healthcare resources. $^{68}$ To address the socioeconomic factors that affect adherence, recommendations include family preparedness, patient health insurance, an uninterrupted supply of medicines, sustainable financing, and reliable medication supply systems. ${ }^{3}$

A similar review suggested that successes achieved from more intensive intervention can be further supported through investments in healthcare systems. ${ }^{12}$ Specifically, healthcare teams or health system-related interventions should include the following: training in the education of patients on the use of medicines, continuous monitoring and re-assessment of treatment-particularly monitoring of adherence - uninterrupted ready availability of information, good patient-provider relationships, monitoring adherence, training in communication skills, and evidencebased selection of medications. ${ }^{12,13}$ In our review, at each intervention level, studies that incorporated multiple means of delivery reported better outcomes. ${ }^{19,20,22-24}$ Thus, to achieve better outcomes, it is essential that future interventions consider multiple intervention delivery methods, including training of healthcare providers.
In this review, fixed-dose therapy interventions were found to be most effective for improving CVD medication adherence. To simplify regimen management, combination or fixed-dose therapy maximizes the number of medicines required while significantly reducing the number of pills a person has to take per time. Providers have a crucial role in optimizing and individualizing the medication regimen, including changing prescriptions from multiple medicines to single-pill, fixed-dose combinations when available.

Team-based care as an intervention to improve medication adherence was found to be particularly effective in our review. ${ }^{19,24,36}$ Physician density is low in most LMICs, further highlighting the need for a team-based care approach to expand access to CVD management. Nurses who work in community health centers or outpatient clinics have considerable access to patients with CVD, among whom they can perform risk assessments. In our review, the nurse-led interventions included patient education and counseling, reminders in the form of nurseinitiated phone interactions and SMS with patients, and a team-based healthcare approach. Similarly, pharmacists delivered efficacious interventions through education, a team-based healthcare approach, and reminders. $^{23,25,29,43,49,54,61}$ It is essential that nurses and pharmacists play a more active role in the development and implementation of medication adherence interventions, particularly at the community level, where they are seen as critical resources.

For interventions that focused on reminders through phone calls and SMS, we found variations in the effectiveness. SMS reminders that were bi-directional and interactive $^{24,34}$ yielded a higher level of adherence and blood pressure control than studies in which the SMS interventions were generic, passive, and one-way. ${ }^{22,69}$ Therefore, in designing an SMS or reminder-based intervention, it is essential to consider personalized, bidirectional, and interactive messages. The messages should be tailored to each patient's needs and timed to coincide with each patient's scheduled medication doses. In this review, many of the reminder-based interventions included using technology in the form of phone call reminders, interactive and informational SMS, and videos. These interventions also have the potential to improve health literacy. There are opportunities for technologydriven interventions in LMIC, for improving the quality of CVD care, medication adherence, and self-care management. $^{68}$ 
Overall, we found a modest body of evidence on the effectiveness of CVD medication adherence interventions in LMICs, as corroborated in similar systematic reviews on medication adherence in LMICs. ${ }^{14}$ However, the effects were inconsistent and varied by study design and country, which has also been found in a similar review. ${ }^{67}$ Many interventions in this review relied on existing healthcare interventions and resources while targeting local factors that affected medication adherence. These interventions can be adapted or adopted to other LMICs according to resource availability.

This review has some limitations. Medication adherence interventions in the studies reviewed were diverse, with different levels of complexity, delivery, and outcome assessments. Hence, we could not substantially categorize the interventions based on the level of intervention complexity nor undertake meta-analysis. Also, as with any systematic review, we acknowledge that some studies may have been missed despite thorough search strategies. Nonetheless, a major strength of this review is that the studies included were distinct in design, and included randomized controlled trials, non-randomized/quasi-experimental studies, and cohort studies. This provides an opportunity to evaluate the external validity of the studies and the extent to which the interventions may be conducted in real-world settings.

Non-adherence to medication is a significant factor in CVD management and control associated with increased risk of poor CVD outcomes and complications. This review shows that comprehensive medication adherence interventions that simultaneously incorporate multiple strategies are effective, especially when the local nuances and contexts such as cost of medicines, availability of infrastructure for technologydependent interventions, health literacy, and beliefs are properly integrated into the delivery of the intervention. This is particularly important for future studies on improving the delivery of medication adherence interventions in LMICs.

\section{Acknowledgments}

This research received no specific grant from any funding agency in the public, commercial, or not-for-profit sectors. We would like to thank Dr. Sarah Szanton for providing feedback and suggestions on improving the methods and synthesis of the results.

\section{Disclosure}

The authors report no conflicts of interest.

\section{References}

1. Bovet P, Paccaud F. Cardiovascular disease and the changing face of global public health: a focus on low and middle middle-incomeies. Public Health Rev. 2011;33(2):397-415. doi:10.1007/BF03391643

2. Bansilal S, Castellano JM, Fuster V. Global burden of CVD: focus on secondary prevention of cardiovascular disease. Int $J$ Cardiol. 2015;201(Suppl 1):S1-S7. doi:10.1016/S0167-5273(15)31026-3

3. World Health Organization. Adherence to Long-Term Therapy: Evidence for Action. World Health Organization; 2003.

4. Kronish IM, Ye S. Adherence to cardiovascular medications: lessons learned and future directions. Prog Cardiovasc Dis. 2013;55(6):590-600.

5. Mills KT, Bundy JD, Kelly TN, et al. Global disparities of hypertension prevalence and control: a systematic analysis of population-based studies from 90 countries. Circulation. 2016;134 (6):441-450. doi:10.1161/CIRCULATIONAHA.115.018912

6. Vrijens B, Vincze G, Kristanto P, Urquhart J, Burnier M. Adherence to prescribed antihypertensive drug treatments: longitudinal study of electronically compiled dosing histories. BMJ. 2008;336 (7653):1114-1117. doi:10.1136/bmj.39553.670231.25

7. Michel B, Egan BM. Adherence in hypertension. Circ Res. 2019;124 (7):1124-1140. doi:10.1161/CIRCRESAHA.118.313220

8. Morrison A, Stauffer ME, Kaufman AS. Defining medication adherence in individual patients. Patient Prefer Adherence. 2015;9:893-897. doi:10.2147/PPA.S86249

9. Brown MT, Bussell JK. Medication adherence: WHO cares? Mayo Clin Proc. 2011;86(4):304-314. doi:10.4065/mcp.2010.0575

10. Ndumele CD, Shaykevich S, Williams D, Hicks LS. Disparities in adherence to hypertensive care in urban ambulatory settings. $J$ Health Care Poor Underserved. 2010;21(1):132-143. doi:10.1353/hpu.0.0259

11. Polinski JM, Kesselheim AS, Frolkis JP, Wescott P, Allen-Coleman C, Fischer MA. A matter of trust: patient barriers to primary medication adherence. Health Educ Res. 2014;29(5):755-763. doi:10.1093/ her/cyu023

12. Kini V, Ho PM. Interventions to improve medication adherence: a review. JAMA. 2018;320(23):2461-2473. doi:10.1001/jama.2018.19271

13. Conn VS, Ruppar TM. Medication adherence outcomes of 771 intervention trials: systematic review and meta-analysis. Prev Med. 2017;99:269-276. doi:10.1016/j.ypmed.2017.03.008

14. Nielsen JØ, Shrestha AD, Neupane D, Kallestrup P. Non-adherence to anti-hypertensive medication in low- and middle-income countries: a systematic review and meta-analysis of 92443 subjects. $J$ Hum Hypertens. 2017;31(1):14-21. doi:10.1038/jhh.2016.31

15. Macquart de Terline D, Kane A, Kramoh KE, et al. Factors associated with poor adherence to medication among hypertensive patients in twelve low and middle income Sub-Saharan countries. PLoS One. 2019;14(7):e0219266. doi:10.1371/journal.pone.0219266

16. Moher D, Liberati A, Tetzlaff J, Altman DG. Preferred reporting items for systematic reviews and meta-analyses: the PRISMA statement. BMJ. 2009;339(jul21 1):b2535. doi:10.1136/bmj.b2535

17. Covidence systematic review software, Veritas Health Innovation, Melbourne, Australia. Available at www.covidence.org.

18. The Joanna Briggs institute critical apprisal tools. Available from: https://jbi.global/critical-appraisal-tools. Accessed February 26, 2020.

19. Adeyemo A, Tayo BO, Luke A, Ogedegbe O, Durazo-Arvizu R, Cooper RS. The Nigerian antihypertensive adherence trial: a community-based randomized trial. $J$ Hypertens. 2013;31 (1):201-207. doi:10.1097/HJH.0b013e32835b0842

20. Odusola A, Nelissen H, Hendriks M, et al. How group-based cardiovascular health education affects treatment adherence and blood pressure control among insured hypertensive Nigerians: a Pre-Test, Post-Test Study. World J Cardiovasc Dis. 2015;5(07):181-198. doi:10.4236/wjed.2015.57021 
21. Sarfo F, Treiber F, Gebregziabher M, et al. PINGS (phone-based intervention under nurse guidance after stroke). Stroke. 2018;49 (1):236-239. doi:10.1161/STROKEAHA.117.019591

22. Bobrow K, Farmer AJ, Springer D, et al. Mobile phone text messages to support treatment adherence in adults with high blood pressure (SMS-text adherence support [StAR]): a single-blind, randomized trial. Circulation. 2016;133(6):592-600. doi:10.1161/CIRCULATI ONAHA.115.017530

23. Aguiar PM, Balisa-Rocha B, Brito GC, Lyra DP Jr. Pharmaceutical care program for elderly patients with uncontrolled hypertension. $J \mathrm{Am}$ Pharm Assoc. 2012;52(4):515-518. doi:10.1331/JAPhA.2012.11015

24. Bonetti AF, Bagatim BQ, Mendes AM, et al. Impact of discharge medication counseling in the cardiology unit of a tertiary hospital in brazil: a randomized controlled trial. Clinics (Sao Paulo). 2018;73: e325. doi:10.6061/clinics/2018/e325

25. Azevedo M, Pedrosa R, Aoqui C, Martins R, Junior T. Effectiveness of home pharmaceutical interventions in metabolic syndrome: a randomized controlled trial. Braz J Pharm Sci. 2017;53. doi:10.1590/s2175-97902017000216089.

26. de Souza AC, Moreira TM, Oliveira ES, et al. Effectiveness of educational technology in promoting quality of life and treatment adherence in hypertensive people. PLoS One. 2016;11(11): e0165311. doi:10.1371/journal.pone.0165311

27. Lourenço LB, Rodrigues RC, Ciol MA, et al. A randomized controlled trial of the effectiveness of planning strategies in the adherence to medication for coronary artery disease. $J$ Adv Nurs. 2014;70 (7):1616-1628. doi:10.1111/jan.12323

28. Mariani J, Rosende A, De Abreu M, et al. Multicap to improve adherence after acute coronary syndromes: results of a randomized controlled clinical trial. Ther Adv Cardiovasc Dis. 2020;14:1753944720912071. doi:10.1177/1753944720912071

29. Morgado M, Rolo S, Castelo-Branco M. Pharmacist intervention program to enhance hypertension control: a randomised controlled trial. Int J Clin Pharm. 2011;33(1):132-140. doi:10.1007/s11096010-9474-x

30. Varleta P, Acevedo M, Akel C, et al. Mobile phone text messaging improves antihypertensive drug adherence in the community. J Clin Hypertens (Greenwich). 2017;19(12):1276-1284. doi:10.1111/ jch.13098

31. Al-Qudah R, Bulatova NR, Obeidat NM, Basheti IA. Impact of home medication management review on medication adherence among Jordanian patients. J Pharm Health Serv Res. 2018;9(3):227-235. doi:10.1111/jphs. 12243

32. Alhalaiqa F, Deane KHO, Nawafleh AH, Clark A, Gray R. Adherence therapy for medication non-compliant patients with hypertension: a randomised controlled trial. J Hum Hypertens. 2012;26(2):117-126. doi:10.1038/jhh.2010.133

33. Farazian F, Emami Moghadam Z, Heshmati Nabavi F, Behnam Vashani H. Effect of self-care education designed based on bandura's self-efficacy model on patients with hypertension: a randomized clinical trial. EBCJ. 2019;9(2):44-52. doi:10.22038/ebcj.2019. 36466.1944

34. Golshahi J, Ahmadzadeh H, Sadeghi M, Mohammadifard N, Pourmoghaddas A. Effect of self-care education on lifestyle modification, medication adherence and blood pressure in hypertensive adults: randomized controlled clinical trial. Adv Biomed Res. 2015;4:204. doi:10.4103/2277-9175.166140

35. Haidari A, Moeini M, Khosravi A. The impact of peer support program on adherence to the treatment regimen in patients with hypertension: a Randomized Clinical Trial Study. Iran J Nurs Midwifery Res. 2017;22 (6):427-430. doi:10.4103/ijnmr.IJNMR_16_16

36. Hosseininasab M, Jahangard-Rafsanjani $Z$, Mohagheghi A, et al. Self-monitoring of blood pressure for improving adherence to antihypertensive medicines and blood pressure control: a randomized controlled trial. Am J Hypertens. 2014;27(11):1339-1345. doi:10.1093/ajh/hpu062
37. Maslakpak MH, Safaie M. A comparison between the effectiveness of short message service and reminder cards regarding medication adherence in patients with hypertension: a randomized controlled clinical trial. Int J Community Based Nurs Midwifery. 2016;4 (3):209-218.

38. Najafi SS, Shaabani M, Momennassab M, Aghasadeghi K. The nurse-led telephone follow-up on medication and dietary adherence among patients after myocardial infarction: a randomized controlled clinical trial. Int J Community Based Nurs Midwifery. 2016;4 (3):199-208.

39. Nayeri ND, Mohammadi S, Razi SP, Kazemnejad A. Investigating the effects of a family-centered care program on stroke patients' adherence to their therapeutic regimens. Contemp Nurse. 2014;47 (1-2):88-96. doi:10.5172/conu.2014.47.1-2.88

40. Yazdanpanah Y, Saleh Moghadam AR, Mazlom SR, Haji Ali Beigloo R, Mohajer S. Effect of an educational program based on health belief model on medication adherence in elderly patients with hypertension. EBCJ. 2019;9(1):52-62. doi:10.22038/ebcj.2019.35215.1895

41. Zakeri MA, Khoshnood Z, Dehghan M, Abazari F. The effect of the continuous care model on treatment adherence in patients with myocardial infarction: a randomised controlled trial. J Res Nurs. 2020;25 (1):54-65. doi:10.1177/1744987119890666

42. Calano BJD, Cacal MJB, Cal CB, et al. Effectiveness of a community-based health programme on the blood pressure control, adherence and knowledge of adults with hypertension: a PRECEDE-PROCEED model approach. $J$ Clin Nurs. 2019;28(9 -10):1879-1888. doi:10.1111/jocn.14787

43. Siang T, Hassali M, Fen N. The role of pharmacist in managing hypertension in the community: findings from a Community Based Study. Indian J Pharm Educ Res. 2019;53(3):553-561. doi:10.5530/ ijper.53.3.88

44. Di M, Mao C, Yang Z, et al. Lack of effects of evidence-based, individualised counselling on medication use in insured patients with mild hypertension in china: a randomised controlled trial. BMJ Evid Based Med. 2019:bmjebm-111197. doi:10.1136/bmjebm-2019-111197

45. Fang R, Li X. Electronic messaging support service programs improve adherence to lipid-lowering therapy among outpatients with coronary artery disease: an Exploratory Randomised Control Study. J Clin Nurs. 2016;25(5-6):664-671. doi:10.1111/jocn.12988

46. Huo X, Krumholz HM, Bai X, et al. Effects of mobile text messaging on glycemic control in patients with coronary heart disease and diabetes mellitus: a randomized clinical trial. Circ Cardiovasc Qual Outcomes. 2019;12(9):e005805. doi:10.1161/CIRCOUTCOMES.119.005805

47. Wan L, Zhang X, You L, Ruan H, Chen S. The efficacy of a comprehensive reminder system to improve health behaviors and blood pressure control in hypertensive ischemic stroke patients: a randomized controlled trial. J Cardiovasc Nurs. 2018;33 (6):509-517. doi:10.1097/JCN.0000000000000496

48. Yu M, Chair SY, Chan CWH, Choi KC. A health education booklet and telephone follow-ups can improve medication adherence, health-related quality of life, and psychological status of patients with heart failure. Heart Lung. 2015;44(5):400-407. doi:10.1016/j. hrtlng.2015.05.004

49. Zhao S, Zhao H, Du S, Qin Y. The impact of clinical pharmacist support on patients receiving multi-drug therapy for coronary heart disease in china: a Long-Term Follow-Up Study. Eur J Hosp Pharm. 2015;22(6):323-327. doi:10.1136/ejhpharm-2014-000632

50. Huang B, Li Z, Wang Y, et al. Effectiveness of self-management support in maintenance haemodialysis patients with hypertension: a pilot cluster randomized controlled trial. Nephrology (Carlton). 2018;23(8):755-763. doi:10.1111/nep.13098

51. Hsu CI, Hsiao FY, Wu FLL, Shen LJ. Adherence and medication utilisation patterns of fixed-dose and free combination of angiotensin receptor blocker/thiazide diuretics among newly diagnosed hypertensive patients: a Population-Based Cohort Study. Int J Clin Pract. 2015;69(7):729-737. doi:10.1111/ijcp.12591 
52. Joshi R, Agrawal T, Fathima F, et al. Cardiovascular risk factor reduction by community health workers in rural India: a cluster-randomized. Am Heart J. 2019;216:9-19. doi:10.1016/j. ahj.2019.06.007

53. Kavita TJS, Vijayvergiya R, Ghai S, Ghai S. Task shifting of cardiovascular risk assessment and communication by nurses for primary and secondary prevention of cardiovascular diseases in a tertiary health care setting of northern India. BMC Health Serv Res. 2020;20(1):10-19. doi:10.1186/s12913-019-4864-9

54. Sundararajan S, Thukani Sathanantham S, Palani S. The effects of clinical pharmacist education on lifestyle modifications of postmyocardial infarction patients in South Isouth ndia: a Prospective Interventional Study. Curr Ther Res Clin Exp. 2020;92:100577. doi:10.1016/j.curtheres.2020.100577

55. Sharma KK, Gupta R, Mathur M, et al. Non-physician health workers for improving adherence to medications and healthy lifestyle following acute coronary syndrome: 24-Month Follow-Up Study. Indian Heart J. 2016;68(6):832-840. doi:10.1016/j.ihj.2016.03.027

56. Sheilini M, Hande HM, Prabhu MM, Pai MS, George A. Impact of multimodal interventions on medication nonadherence among elderly hypertensives: a Randomized Controlled Study. Patient Prefer Adherence. 2019;13:549-559. doi:10.2147/PPA.S195446

57. Xavier D, Gupta R, Kamath D, et al. Community health worker-based intervention for adherence to drugs and lifestyle change after acute coronary syndrome: a multicentre, open, randomised controlled trial. Lancet Diabetes Endocrinol. 2016;4(3):244-253. doi:10.1016/S2213-8587(15)00480-5

58. Nguyen HL, Allison JJ, Ha DA, et al. Culturally adaptive storytelling intervention versus didactic intervention to improve hypertension control in vietnam: a cluster-randomized controlled feasibility trial. Pilot Feasibility Stud. 2017;3(1):22. doi:10.1186/s40814-017-0136-9

59. Kamal AK, Khalid W, Muqeet A, et al. Making prescriptions "talk" to stroke and heart attack survivors to improve adherence: results of a randomized clinical trial (The Talking $\mathrm{Rx}$ Study). PLoS One. 2018;13(12):e0197671. doi:10.1371/journal.pone.0197671

60. Kamal AK, Shaikh Q, Pasha O, et al. A randomized controlled behavioral intervention trial to improve medication adherence in adult stroke patients with prescription tailored short messaging service (SMS)-SMS4Stroke study. BMC Neurol. 2015;15(1):212. doi:10.1186/s12883-015-0471-5
61. Saleem F, Hassali MA, Shafie AA, et al. Pharmacist intervention in improving hypertension-related knowledge, treatment medication adherence and health-related quality of life: a non-clinical randomized controlled trial. Health Expect. 2015;18(5):1270-1281. doi:10.1111/hex.12101

62. Woodham NS, Taneepanichskul S, Somrongthong R, Kitsanapun A, Sompakdee B. Effectiveness of a multidisciplinary approach intervention to improve blood pressure control among elderly hypertensive patients in rural Thailand: a Quasi-Experimental Study. $J$ Multidiscip Healthc. 2020;13:571-580. doi:10.2147/JMDH. S254286

63. Tankumpuan T, Anuruang S, Jackson D, Hickman LD, DiGiacomo M, Davidson PM. Improved adherence in older patients with hypertension: an observational study of a community-based intervention. Int $J$ Older People Nurs. 2019;14(3):e12248. doi:10.1111/opn.12248

64. Chiu CW, Wong FKY. Effects of 8 weeks sustained follow-up after a nurse consultation on hypertension: a randomised trial. Int J Nurs Stud. 2010;47(11):1374-1382. doi:10.1016/j.ijnurstu.2010.03.018

65. Park J, Shin Y, Lee S, Lee SI. Antihypertensive drug medication adherence and its affecting factors in South Korea. Int $J$ Cardiol. 2008;128(3):392-398. doi:10.1016/j.ijcard.2007.04.114

66. Costa E, Giardini A, Savin M, et al. Interventional tools to improve medication adherence: review of literature. Patient Prefer Adherence. 2015;9:1303-1314. doi:10.2147/PPA.S87551

67. Nieuwlaat R, Wilczynski N, Navarro $\mathrm{T}$, et al. Interventions for enhancing medication adherence. Cochrane Database Syst Rev. 2014;(11):CD000011. doi:10.1002/14651858.CD000011.pub4

68. Bosworth HB, Granger BB, Mendys P, et al. Medication adherence: a call for action. Am Heart J. 2011;162(3):412-424. doi:10.1016/j. ahj.2011.06.007

69. Roshandel G, Khoshnia M, Poustchi H, et al. Effectiveness of polypill for primary and secondary prevention of cardiovascular diseases (PolyIran): a pragmatic, cluster-randomised trial. Lancet. 2019;394 (10199):672-683. doi:10.1016/S0140-6736(19)31791-X
Patient Preference and Adherence

\section{Publish your work in this journal}

Patient Preference and Adherence is an international, peer-reviewed, open access journal that focusing on the growing importance of patient preference and adherence throughout the therapeutic continuum. Patient satisfaction, acceptability, quality of life, compliance, persistence and their role in developing new therapeutic modalities and compounds to optimize clinical outcomes for existing disease states are major areas of interest for the journal. This journal has been accepted for indexing on PubMed Central. The manuscript management system is completely online and includes a very quick and fair peer-review system, which is all easy to use. Visit http:/ www.dovepress.com/testimonials.php to read real quotes from published authors. 